H. Heyer

Nagoya Math. J.

Vol. 116 (1989), 43-62

\title{
INFINITELY DIVISIBLE PROBABILITY MEASURES ON A DISCRETE GELFAND PAIR
}

\author{
H. HEYER
}

\section{Dedicated to Professor Hida on the occasion of his 60th birthday}

\section{§1. Introduction}

Infinitely divisible probability measures on discrete spaces admitting a commutative convolution have been studied in various frameworks. For generalized convolutions related to Delphic structures an important contribution was made by Gilewski and Urbanik in [5]. In [11] Schwartz based his detailed analysis on convolutions arising from orthogonal series. Both of these approaches can be included into the framework of discrete hypergroups chosen f.e. by Gallardo and Gebuhrer in [4]. The main result common to these sources is the fact that, roughly speaking, all infinitely divisible probability measures are of Poisson type. Although the double coset spaces of Gelfand pairs are commutative hypergroups admitting an extended harmonic analysis, the analytic methods developed in the theory of Gelfand pairs (see Dieudonné [3] and Heyer [7]) provide a more direct access to specific results like the characterization of divisible (idempotent, infinitely divisible) measures. For discrete Gelfand pairs (and their double coset spaces) Letac and his school have achieved remarkable results (see Letac [8], [9] and the references therein). The still unpublished thesis [1] of S. Ben Mansoor contains much information about divisible probability measures on cubes. In our exposition we reprove Ben Mansoor's main theorem 3.5.4 with additional care, and discuss the special case of the $m$-dimensional cube which was the basic object of study already in Letac, Takács [10]. It turns out that the main obstacle to be overcome in establishing the Poisson representation of infinitely divisible probability measures is the presence of idempotent factors, a problem that has been excluded f.e. in the work [4] of Gallardo and Gebuhrer.

In the following presentation we aim at a selfcontained analysis of

Received November 28, 1987. 
the problems of idempotency and infinite divisibility which stresses some of the technical points neglected in previous publications. This concerns the sections 4 and 5 . Sections 2 and 3 contain preliminaries on the method of Gelfand pairs and spherical functions along the standard references Dieudonné [3] and Bougerol [2]. For the theory of probability measures on locally compact groups the reader is referred to parts of the author's monograph [6]. It is the author's pleasure to pay tribute to K. Hunzinger who in his Diploma thesis struggled successfully with some of the sources.

\section{§2. Generalities on Gelfand pairs}

Let $G$ be a multiplicatively written group and let $T$ be an arbitrary set. The pair $(G, T)$ is said to constitute a homogeneous space if the mapping $(g, t) \rightarrow g(t)$ from $G \times T$ into $T$ has the following properties: (i) For the neutral element $e$ of $G$ one has $e(t)=t$ for all $t \in T$ (ii) For all $g, h \in G$ and $t \in T, g h(t)=g(h(t))$, and (iii) For every pair $(t, s) \in T \times T$ there exists a $g \in G$ such that $g(t)=s$. For any fixed $t \in T$ the set $\{g \in G: g(t)=t\}$ is called the stabilizer of $t$. Note that given a homogeneous space $(G, T)$, for any $g \in G$ the mapping $t \rightarrow g(t)$ from $T$ into $T$ is a bijection.

Let $(G, T)$ be a homogeneous space, $t_{0} \in T$ and $K$ the stabilizer of $t_{0}$. Then $K$ is a subgroup of $G$, and the mapping $g k \rightarrow g\left(t_{0}\right)$ from $G / K$ into $T$ is a bijection. There is an equivalence relation defined on $T \times T$ by the requirement that given $(t, s)$ and $\left(t^{\prime}, s^{\prime}\right) \in T \times T$ there exists a $g \in G$ such that $g(t)=t^{\prime}$ and $g(s)=s^{\prime}$. The class associated with $(t, s) \in T \times T$ will be abbreviated by $x(t, s)$. The class defined by $x(t, t)=\left(t^{\prime}, t^{\prime}\right)$ for all $t, t^{\prime} \in T$ is denoted by $x_{0}$. Finally we introduce the set $X=\{x(t, s):(t, s)$ $\in T \times T\}$ of all equivalence classes $x(t, s)$ associated with $(t, s) \in T \times T$. Clearly $X$ can be identified with the set $G \| K$ of double coset classes $K g K(g \in G)$ of $G$ with respect to $K$. More precisely, given $(G, T), t_{0}$ and $K$ as above, for every $t \in T$ we have $X=\{x(t, g(t)): g \in G\}$, and the mapping $x\left(t_{0}, g\left(t_{0}\right)\right) \rightarrow K g K$ from $X$ into $G / K$ is a bijection. Note that for any subgroup $K$ of $G$ the pair $(G, G / K)$ constitutes a homogeneous space, with the $G$-action on $G / K$ given by $(g, h K) \rightarrow g h K$ for all $g, h \in G$.

Now let $\mathscr{T}$ denote a topology on $G$ such that $(G, \mathscr{T})$ becomes a locally compact, unimodular, separable and metrizable group and that $K$ is a compact subgroup of $G$. It is known that by this choice of $\mathscr{T} G / K$ is a 
locally compact homogeneous space admitting an essentially unique $G$ invariant measure $\pi \geqq 0$. By the identification $G / K \leftrightarrow T$ we therefore obtain an essentially unique $G$-invariant measure $\pi \geqq 0$ on $T$. Introducing in $X$ the final topology with respect to the mapping $\phi=\phi_{t_{0}}: t \rightarrow x\left(t_{0}, t\right)$ from $T$ into $X$, we end up with a measure $\omega:=\phi(\pi) \geqq 0$ on $X$.

Let $x \in X$ and let $(t, s) \in T \times T$ be such that $x=x(t, s)$. For any $a$, $b \in L^{1}(X, \omega)$ the number

$$
a * b(x):=\int a(x(t, r)) b(x(r, s)) \pi(d r)
$$

is well-defined, the convolution $a * b$ of $a$ and $b$ lies in $L^{1}(X, \omega)$, and we have

$$
\int a * b d \omega=\int a d \omega \int b d \omega
$$

Although this convolution in $L^{1}(X, \omega)$ is associative, in general it is neither commutative nor admits a unit in $L^{1}(X, \omega)$. If however, $x(t, s)=$ $x(s, t)$ for all $(t, s) \in T \times T$, convolution in $L^{1}(X, \omega)$ is a commutative operation. Note that the convolution can be extended to the space $L^{1}(X, \omega) \cup$ $L^{\infty}(X, \omega)$. For $a \in L^{1}(X, \omega)$ and $b \in L^{1}(X, \omega) \cup L^{\infty}(X, \omega)$ we have

$$
a * b\left(x_{0}\right)=\int a \check{b} d \omega
$$

where $\check{b}$ is defined by

$$
\check{b}\left(x\left(t, t_{0}\right)\right):=b\left(x\left(t_{0}, t\right)\right) \quad \text { for all } t \in T .
$$

The pair $(G, K)$ is called a Gelfand pair if the convolution in $L^{1}(X, \omega)$ is commutative. A Gelfand pair $(G, K)$ is said to be discrete or finite if $T \leftrightarrow G / K$ is countable or finite. A Gelfand pair $(G, K)$ is said to be symmetric if for all $(t, s) \in T \times T$ one has

$$
x(t, s)=x(s, t) .
$$

If $T$ is at most countable, $t_{0} \in T, K$ is the stabilizer of $t_{0}$ and $(G, K)$ a Gelfand pair, then the discrete topology on $T$ and on $X$ permits us to choose $\pi$ as the counting measure on $T$. In this particular case

$$
\omega(x)=\left|\left\{t \in T: x\left(t_{0}, t\right)=x\right\}\right|
$$

for all $x \in X$. 


\section{§3. Spherical functions}

Let $(G, K)$ be a Gelfand pair arising from a homogeneous space $(G, T)$, a $t_{0} \in T$ and the stabilizer $K$ of $t_{0}$. A function $\phi \in L^{\infty}(X, \omega)$ with $\phi \neq \equiv$ is said to be spherical for $(G, K)$ if for all $a, b \in L^{1}(X, \omega)$ one has

$$
a * b * \phi\left(x_{0}\right)=a * \phi\left(x_{0}\right) \cdot b * \phi\left(x_{0}\right) .
$$

By $S(G, K)$ we denote the totality of spherical functions for $(G, K)$. Clearly $1_{x} \in S(G, K)$. It is easily checked that according to

3.1 Proposition for any $\phi \in L^{\infty}(X, \omega)$ the following statements are equivalent:

(i) $\phi \in S(G, K)$.

(ii) $\phi\left(x_{0}\right)=1$ and $a * \phi=a * \phi\left(x_{0}\right) \cdot \phi$ for all $a \in L^{1}(X, \omega)$.

In particular, spherical functions for $(G, K)$ are pairwise linearly independent.

If $(G, K)$ is a discrete Gelfand pair, then $L^{1}(X, \omega)$ becomes a separable commutative Banach algebra with unit element $1_{x_{0}}$.

For any Gelfand pair $(G, K)$ we define for a given function $a \in L^{1}(X, \omega)$ the mapping $e_{a}: L^{\infty}(X, \omega) \rightarrow L^{\infty}(X, \omega)$ given by

$$
e_{a}(b):=a * b \quad \text { for all } b \in L^{\infty}(X, \omega) .
$$

The above characterization of spherical functions yields immediately that for every $a \in L^{1}(X, \omega)$ the set $\left\{a * \phi\left(x_{0}\right): \phi \in S(G, K)\right\}$ contains only eigenvalues of $e_{a}$. In the special case of a discrete Gelfand pair $(G, K)$ we see that given $a \in L^{1}(X, \omega),\left\{a * \phi\left(x_{0}\right): \phi \in S(G, K)\right\}$ is exactly the set of eigenvalues of $e_{a}$. As a consequence we obtain the fact that $S(G, K)$ is a basis of the subspace of $L^{\infty}(X, \omega)$ which is spanned by all eigenvectors of $e_{a}$.

We shall discuss the case of a finite Gelfand pair in more detail. For any finite Gelfand pair $(G, K)$ we have

$$
L^{1}(X, \omega)=L^{p}(X, \omega)
$$

whenever $p \in \bar{N}$. In particular, we obtain a scalar product $\langle\cdot, \cdot\rangle$ in $L^{1}(X, \omega)$ defined by

$$
\langle a, b\rangle:=a * \check{\check{b}}\left(x_{0}\right)
$$

for all $a, b \in L^{1}(X, \omega)$. With this scalar product $L^{1}(X, \omega)$ becomes a Hilbert space. Note that any $\phi \in S(G, K)$ satisfies $\phi=\check{\phi}$. 
3.2 Proposition.

(1) The set $\left\{\frac{1}{{ }_{+} \sqrt{\langle\phi, \phi\rangle}}: \phi \in S(G, K)\right\}$ forms $a$

Hilbert basis of $L^{1}(X, \omega)$, and

(2) any $a \in L^{1}(X, \omega)$ admits a representation

$$
a=\sum_{\phi \in S(G, K)} \frac{\langle a, \phi\rangle}{\langle\phi, \phi\rangle} \cdot s .
$$

Returning to an arbitrary discrete Gelfand pairs $(G, K)$ we introduce the dual $Z(G, K)$ of $(G, K)$ as the set of spherical functions $\phi \in S(G, K)$ which are positive definite in the following sense: For every $n \geqq 1$, all choices $\lambda_{1}, \cdots, \lambda_{n} \in C$ and $t_{1}, \cdots, t_{n} \in T$

$$
\sum_{i, j=1}^{n} \lambda_{i} \bar{\lambda}_{j} \phi\left(x\left(t_{i}, t_{j}\right)\right) \geqq 0 .
$$

Note that if the pair $(G, K)$ is also symmetric then any function $a$ on $X$ satisfies $a=\check{a}$, and any positive definite $a$ on $X$ satisfies even $a=\bar{a}$.

Let us now consider the weak topology $\sigma\left(L^{\infty}, L^{1}\right)$ in $L^{\infty}(X, \omega)$. It turns out that $S(G, K)$ is $\sigma\left(L^{\infty}, L^{1}\right)$-compact. Since $Z(G, K)$ is $\sigma\left(L^{\infty}, L^{1}\right)$-closed in $S(G, K)$, it is also compact. An application of the Plancherel-Godement theorem (Dieudonné [3], 22.7.5) for Gelfand pairs yields the

3.3 Proposition. There exists a unique probability measure $\sigma$ on $Z(G, K)$ such that any $a \in L^{1}(X, \omega)$ admits the representation

$$
a(x)=\int a * \phi\left(x_{0}\right) \check{\phi}(x) \sigma(d \phi)
$$

valid for all $x \in X$.

The measure $\sigma$ is called the Plancherel measures of the Gelfand pair $(G, K)$.

We observe that whenever, given $a, b \in L^{1}(X, \omega)$,

$$
a * \phi\left(x_{0}\right)=b * \phi\left(x_{0}\right)
$$

for all $\phi \in Z(G, K)$, then $a=b$.

\section{§4. Idempotency}

Given a discrete Gelfand pair $(G, K)$ with the corresponding spaces $T \cong G / K$ and $X=G / / K$ and their canonical measures $\pi$ and $\omega$ respectively. The spaces of ( $\omega$-absolutely continuous) bounded or probability measures 
on $X$ will be identified with the function spaces $L^{1}(X, \omega)$ and

$$
M^{1}(X, \omega):=\left\{\mu \in L^{1}(X, \omega): \mu \geqq 0,\|\mu\|_{1}=1\right\}
$$

respectively. The essentially unique (left) invariant measure on $G$ will be abbreviated by $\omega_{G}$.

We recall that a measure $\mu \in M^{1}(X, \omega)$ is called an idempotent if $\mu^{2}=\mu$.

4.1 Theorem. For every idempotent $\lambda \in M^{1}(X, \omega)$ there exists a compact subgroup $K_{\lambda}$ with the following properties:

(i) $K$ is a subgroup of $K_{\lambda}$.

(ii) $\omega_{G}\left(K_{\lambda}\right)=\left|\left\{g\left(t_{0}\right): g \in K_{\lambda}\right\}\right|$.

(iii) $\lambda\left(x\left(t_{0}, t\right)\right)= \begin{cases}\omega_{G}\left(K_{\lambda}\right)^{-1} & \text { if there is a } g \in K_{\lambda} \text { such that } g\left(t_{0}\right)=t \\ 0 & \text { otherwise. }\end{cases}$

Proof. Let $\lambda \in M^{1}(X, \omega)$ be an idempotent. Then $\lambda\left(x_{0}\right) \geqq \lambda(x) \grave{\lambda}(x) \geqq 0$ for all $x \in X$ and $\lambda\left(x_{0}\right)>0$. Defining $\lambda_{1}(g):=\lambda\left(x\left(t_{0}, g\left(t_{0}\right)\right)\right.$ for all $g \in G$ we obtain an idempotent $\lambda_{1} \in L_{+}^{1}\left(G, \omega_{G}\right)$. By a well-known result (Heyer [6], 1.2.10) there exists a compact subgroup $K_{\lambda}$ of $G$ such that $\lambda_{1} \cdot \omega_{G}=\omega_{K_{\lambda}}$ where, as usual, $\omega_{K_{\lambda}}$ denotes the normed Haar measure of $K_{\lambda}$. Clearly, $0<\omega_{G}\left(K_{\lambda}\right)<\infty$. Thus, the measure $\omega_{G}\left(K_{\lambda}\right)^{-1} 1_{K_{\lambda}} \cdot \omega_{G}$ is a Haar measure on $K_{2}$. Consequently $\lambda_{1}=\omega_{G}\left(K_{\lambda}\right)^{-1} 1_{K_{\lambda}}\left[\omega_{G}\right]$, and (iii) follows. Since

$$
0<\lambda\left(x_{0}\right)=\lambda_{1}(g)=\omega_{G}\left(K_{\lambda}\right)^{-1} 1_{K_{\lambda}}(g)
$$

for all $g \in K, 1_{K_{\lambda}}(g)=1$ for all $g \in K$, and we have (i). (iii) together with the equality

$$
\sum_{t \in T} \lambda\left(x\left(t_{0}, t\right)\right)=1
$$

implies (ii).

4.2 Corollary. For every idempotent $\lambda \in M^{1}(X, \omega)$ there exists a subset $A$ of $X$ such that

$$
\lambda=\lambda\left(x_{0}\right) 1_{A}
$$

Proof. Let $\lambda \in M^{1}(X, \omega)$ be an idempotent. From the theorem we obtain a subgroup $K_{\lambda}$ of $G$ satisfying

$$
\lambda\left(x\left(t_{0}, t\right)\right)= \begin{cases}\omega_{G}\left(K_{\lambda}\right)^{-1} & \text { if } g\left(t_{0}\right)=t \text { for some } g \in K_{\lambda} \\ 0 & \text { otherwise }\end{cases}
$$

Putting 


$$
A:=\left\{x\left(t_{0}, t\right): \text { There is a } g \in K \text { with } g\left(t_{0}\right)=t\right\}
$$

we observe that $x_{0} \in A$ and that

$$
\lambda(x)= \begin{cases}\lambda\left(x_{0}\right) & \text { if } x \in A \\ 0 & \text { if } x \notin A\end{cases}
$$

4.3. Discussion. Given $\lambda \in M^{1}(X, \omega)$ and $K_{\lambda}$ as above we introduce the sets

and

$$
T_{\lambda}:=\left\{g\left(t_{0}\right): g \in K_{\lambda}\right\}
$$

$$
T^{\lambda}:=\left\{g\left(T_{\lambda}\right): g \in G\right\} .
$$

Then $\left(G, T^{\lambda}\right)$ becomes a homogeneous space with corresponding double coset space $X_{\lambda}$. For every $x_{\lambda} \in X_{\lambda}$ we define the number

$$
\omega_{\lambda}\left(x_{\lambda}\right):=\mid\left\{g\left(T_{\lambda}\right): g \in G \text { and } x_{\lambda}\left(T_{\lambda}, g\left(T_{\lambda}\right)\right)=x\right\} \mid
$$

and obtain a measure $\omega_{\lambda}$ on $X_{\lambda}$. Clearly, there is the natural identification

$$
X_{\lambda} \longleftrightarrow G \| K_{\lambda} .
$$

Since $K$ is a subgroup of $K_{\lambda}$, every $x \in X$ lies in exactly one double coset class $x_{\lambda} \in X_{\lambda}$. Hence, given $\tilde{\nu} \in L^{1}\left(X_{\lambda}, \omega_{\lambda}\right)$ there is a $\tilde{\nu} \in L^{1}(X, \omega)$ defined by

$$
\nu(x):=N_{\lambda}^{-1} \tilde{\nu}\left(x_{\lambda}\right)
$$

for all $x \subset X_{\lambda}, x_{\lambda} \in X_{\lambda}$, where

$$
N_{\lambda}:=\left|\left\{g\left(t_{0}\right): g \in K_{\lambda}\right\}\right| .
$$

For the convolution in $L^{1}\left(X_{k}, \omega_{\lambda}\right)$ we shall use the symbol $*_{\lambda}$. The following

4.4. Formula is easily established: For all $\mu, \nu \in L^{1}\left(X_{\lambda}, \omega_{\lambda}\right)$ we have

$$
\tilde{\mu} *_{\lambda} \tilde{\nu}=\widetilde{\mu * \nu} .
$$

In fact, if $T_{\lambda}^{\prime} \in T^{\lambda}$ and $t^{\prime} \in T_{\lambda}^{\prime}$ then

$$
\begin{aligned}
\tilde{\mu} *_{\lambda} \tilde{\nu}\left(x_{\lambda}\left(T_{\lambda}, T_{\lambda}^{\prime}\right)\right) & =\sum_{T_{\lambda}^{\prime \prime} \in T^{\lambda}} \mu\left(x_{\lambda}\left(T_{\lambda}, T_{\lambda}^{\prime \prime}\right)\right) \nu\left(x_{\lambda}\left(T_{\lambda}^{\prime \prime}, T_{\lambda}^{\prime}\right)\right) \\
& =\sum_{T_{\lambda}^{\prime} \in T^{\lambda}} \frac{1}{N_{\lambda}} \sum_{t \in T_{\lambda}^{\prime \prime}} N_{\lambda}^{2} \tilde{\mu}\left(x\left(t_{0}, t\right)\right) \tilde{\nu}\left(x\left(t, t^{\prime}\right)\right) \\
& =N_{\lambda} \sum_{t \in T} \mu\left(x\left(t_{0}, t\right)\right) \nu\left(x\left(t, t^{\prime}\right)\right) \\
& =N_{\lambda \mu} \mu \nu\left(x\left(t_{0}, t^{\prime}\right)\right)=\widetilde{\mu * \nu}\left(x_{\lambda}\left(T_{\lambda}, T_{\lambda}^{\prime}\right)\right) .
\end{aligned}
$$




\subsection{Consequences.}

4.5.1. $\left(G, K_{\lambda}\right)$ is a discrete Gelfand pair.

4.5.2. $\left(G, K_{\lambda}\right)$ is symmetric whenever $(G, K)$ is.

The next result concerns the relationship between the sets $S:=S(G, K)$ and $S_{\lambda}:=S\left(G, K_{\lambda}\right)$ by means of the set

$$
A_{\lambda}:=\left\{\nu \in L^{1}(X, \omega): \lambda * \nu=\nu\right\} .
$$

4.6 Theorem. The mapping $\tilde{\mu} \rightarrow \mu$ from $L^{1}\left(X_{\lambda}, \omega_{\lambda}\right)$ into $L^{1}(X, \omega)$ is a norm-preserving algebra isomorphism from $L^{1}\left(X_{\lambda}, \omega_{\lambda}\right)$ onto $A_{\lambda}$ satisfying

$$
\left\{N_{\lambda} \cdot \phi: \tilde{\phi} \in S_{\lambda}\right\}=A_{\lambda} \cap S .
$$

Proof. 1. Clearly, $A_{\lambda}$ is a subalgebra of $L^{1}(X, \omega)$. From the definition follows directly, that the mapping $\tilde{\mu} \rightarrow \mu$ is injective. The fact that it is an algebra homomorphism becomes obvious from Formula 4.4. In order to obtain the first statement of the theorem it remains to show that $\tilde{\mu} \rightarrow \mu$ is surjective. Let $\nu \in A_{\lambda}$. Then, for all $g_{1}, g_{2} \in K$ and $t \in T$ we have

$$
\begin{aligned}
\nu\left(x\left(g_{1}\left(t_{0}\right), g_{2}(t)\right)\right) & =\lambda * \nu\left(g_{1}\left(t_{0}\right), g_{2}(t)\right) \\
& =\sum_{t^{\prime} \in T} \lambda\left(x\left(g_{1}\left(t_{0}\right), t^{\prime}\right) \nu\left(x\left(t^{\prime}\right), g_{2}(t)\right)\right) \\
& =\sum_{t^{\prime} \in T} \lambda\left(x\left(t_{0}, g_{1}^{-1} g_{2}\left(t^{\prime}\right)\right)\right) \nu\left(x\left(t^{\prime}, t\right)\right) .
\end{aligned}
$$

By Theorem 4.1 it suffices in this last expression to sum only over those $t^{\prime} \in T$ for which $g_{1}^{-1} g_{2}\left(t^{\prime}\right) \in T_{\lambda}$. But the hypothesis implies $g_{1}^{-1} g_{2} \in K_{\lambda}$, hence $g_{2}^{-1} g_{1}\left(T_{\lambda}\right)=T_{\lambda}$ and therefore

$$
\begin{aligned}
\nu\left(x\left(g_{1}\left(t_{0}\right), g_{2}(t)\right)\right) & =\sum_{t^{\prime} \in T_{\lambda}} \lambda\left(x\left(t_{0}, t^{\prime}\right)\right) \nu\left(x\left(t^{\prime}, t\right)\right) \\
& =\sum_{t^{\prime} \in T} \lambda\left(x\left(t_{0}, t^{\prime}\right)\right) \nu\left(x\left(t^{\prime}, t\right)\right) \\
& =\lambda * \nu\left(x\left(t_{0}, t\right)\right) \\
& =\nu\left(x\left(t_{0}, t\right)\right) .
\end{aligned}
$$

Now we choose for each $T_{\lambda}^{\prime} \in T^{\lambda}$ a $t_{\lambda} \in T_{\lambda}^{\prime}$ and put

$$
\tilde{\nu}\left(x_{\lambda}\left(T_{\lambda}, T_{\lambda}^{\prime}\right)\right):=N_{\lambda} \cdot \nu\left(x\left(t_{0}, t_{\lambda}\right)\right)
$$

whenever $T_{\lambda}^{\prime} \in T^{\lambda}$. Then $\tilde{\nu} \in L^{1}\left(X_{\lambda}, \omega_{\lambda}\right)$ and by the above computation, $\nu=\lambda$. I.e. $\tilde{\mu} \rightarrow \mu$ is surjective onto $A_{\lambda}$.

In order to see that this mapping is also norm-preserving we note that for all $\tilde{\mu} \in L^{1}\left(X_{\lambda}, \omega_{2}\right)$ the following equalities hold: 


$$
\begin{aligned}
\int \tilde{\mu} d \omega_{\lambda} & =\sum_{T_{\lambda}^{\prime} \in T^{\lambda}} \tilde{\mu}\left(x_{\lambda}\left(T_{\lambda}, T_{\lambda}^{\prime}\right)\right) \\
& =\sum_{T_{\lambda}^{\prime} \in T^{\prime}} \frac{1}{N_{\lambda}} \sum_{t \in T_{\lambda}^{\prime}} N_{\lambda} \cdot \mu\left(t_{0}, t\right) \\
& =\sum_{t \in T} \mu\left(t_{0}, t\right) \\
& =\int \mu d \omega .
\end{aligned}
$$

2a. We shall prove the inclusion

$$
\left\{N_{\lambda} \cdot \phi: \tilde{\phi} \in S_{\lambda}\right\} \subset A^{\lambda} \cap S .
$$

Let $\tilde{\phi} \in S_{\lambda}$. Then, clearly, $N_{\lambda} \cdot \phi \in A_{\lambda}$ and hence it suffices to prove that $N_{\lambda} \cdot \phi \in S$. If $N_{\lambda} \cdot \phi \in A$ then also $\phi \in A_{\lambda}$. Thus for all $\mu \in L^{1}(X, \omega)$ we have that $\mu * \lambda$ and $\mu * \phi \in A_{\lambda}$ and therefore that $\widetilde{\mu * \lambda}$ and $\widetilde{\mu * \phi}$ are well-defined. Applying Formula 4.4 and Proposition 3.1 we get

$$
\begin{aligned}
\widetilde{\mu * \phi} & =\widetilde{\mu * \lambda * \phi} \\
& =\widetilde{\mu * \lambda} *_{2} \tilde{\phi} \\
& =\widetilde{\mu * \lambda * *_{\lambda}} \tilde{\phi}\left(T_{\lambda}, T_{\lambda}\right) \cdot \tilde{\phi} \\
& =\widetilde{\mu * \lambda * \phi}\left(T_{\lambda}, T_{2}\right) \cdot \tilde{\phi} \\
& =\widetilde{\mu * \phi}\left(T_{\lambda}, T_{\lambda}\right) \cdot \tilde{\phi} \\
& =N_{\lambda} \mu * \phi\left(x_{0}\right) \cdot \tilde{\phi},
\end{aligned}
$$

hence

$$
N_{\lambda} \mu * \phi=N_{\lambda}^{2} \mu * \phi\left(x_{0}\right) \cdot \phi
$$

and therefore

$$
\mu *\left(N_{\lambda} \cdot \phi\right)=\left(\mu *\left(N_{\lambda} \cdot \phi\right)\right)\left(x_{0}\right) \cdot\left(N_{\lambda} \cdot \phi\right)
$$

Since

$$
N_{\lambda} \cdot \phi\left(x_{0}\right)=\tilde{\phi}\left(x_{\lambda}\left(T_{\lambda}, T_{\lambda}\right)\right)=1,
$$

another application of Proposition 3.1 implies that $N_{\lambda} \cdot \phi \in S$.

2 b. It remains to be shown that

$$
A_{\lambda} \cap S \subset\left\{N_{\lambda} \cdot \phi: \tilde{\phi} \in S_{\lambda}\right\}
$$

Let $\phi \in A_{2} \cap S$. By the surjectivity of $\tilde{\mu} \rightarrow \mu$ there exists a $\tilde{\phi}$ in $L^{1}\left(X_{2}, \omega_{2}\right)$, and for each $\tilde{\mu} \in L^{1}\left(X_{\lambda}, \omega_{\lambda}\right)$ we obtain from Proposition 3.1 that 


$$
\begin{aligned}
\tilde{\mu} *_{\lambda}\left(\frac{1}{N_{\lambda}} \cdot \tilde{\phi}\right) & =\frac{1}{N_{\lambda}} \cdot \widetilde{\mu * \phi} \\
& =\frac{1}{N_{\lambda}} \cdot \widetilde{\mu * \phi}\left(x_{0}\right) \cdot \tilde{\phi} \\
& =\tilde{\mu} *_{\lambda}\left(\frac{1}{N_{\lambda}} \cdot \tilde{\phi}\right)\left(x_{\lambda}\left(T_{\lambda}, T_{\lambda}\right)\right) \cdot\left(\frac{1}{N_{\lambda}} \cdot \tilde{\phi}\right) .
\end{aligned}
$$

Since

$$
\left(\frac{1}{N_{\lambda}} \cdot \tilde{\phi}\right)\left(x_{\lambda}\left(T_{\lambda}, T_{\lambda}\right)\right)=\frac{1}{N_{\lambda}} \cdot N_{\lambda} \cdot \phi\left(x_{0}\right)=1
$$

another appeal to Proposition 3.1 implies the desired inclusion.

4.7 Terminology. A measure $\mu \in M^{1}(X, \omega)$ is said to divide another measure $\nu \in M^{1}(X, \omega)$ (in symbols $\mu / \nu$ ) if there exists a measure $\rho \in M^{1}(X, \omega)$ such that

$$
\mu * \rho=\nu \text {. }
$$

By $D(\nu)$ we denote the set of idempotent factors of $\nu$. A measure $\mu \in D(\nu)$ is called a maximal idempotent factor of $\nu$ if

$$
\mu * \rho=\mu
$$

for all $\rho \in D(\nu)$. Finally $\nu \in M^{1}(X, \omega)$ lacks idempotent factors if $D(\nu)=\left\{1_{x_{0}}\right\}$.

\subsection{Remarks.}

4.8.1. Let $\lambda$ be an idempotent in $M^{1}(X, \omega)$ and $\mu \in M^{1}(X, \omega)$ such that $\lambda \mid \mu$. Then there exists a measure $\nu \in M^{1}(X, \omega)$ satisfying $\mu=\lambda * \nu$ and consequently

$$
\mu=\lambda * \nu=\lambda * \lambda * \nu=\lambda * \mu .
$$

4.8.2. For every $\mu \in M^{1}(X, \omega)$ there exists a maximal idempotent factor of $\mu$.

The proof of this last statement is achieved by applying Zorn's Lemma.

\section{$\S 5$. Infinite divisibility}

We start with a discrete Gelfand pair $(G, K)$ having dual space $Z:=Z(G, K)$ and Plancherel measure $\sigma$. For the entire section we suppose that the spherical function $\phi_{0}:=1_{X}$ belongs to $\operatorname{supp}(\sigma)$. This Godement property (GP) implies the validity of 
5.1 Lévy's continuity Theorem (Gallardo, Gebuhrer [4], Théorème 3.1). For any sequence $\left(\mu_{n}\right)_{n \geqq 1}$ of measures in $M^{1}(X, \omega)$ such that there exists a complex-valued function $\Phi$ on $Z$, continuous at $\phi_{0}$ and satisfying

$$
\lim _{n \rightarrow \infty} \mu_{n} * \phi\left(x_{0}\right)=\Phi(\phi)
$$

whenever $\phi \in Z$, there is a measure $\mu \in M^{1}(X, \omega)$ such that

$$
\lim _{n \rightarrow \infty} \mu_{n}(x)=\mu(x)
$$

for all $x \in X$.

There is a variety of Gelfand pairs admitting (GP), f.e. all pairs $(G, K)$ where $G$ is compact. But there are also examples of Gelfand pairs without (GP), f.e. the pair associated with a homogeneous tree.

We recall that a measure $\mu \in M^{1}(X, \omega)$ is said to be infinitely divisible if for every $n \geqq 1$ there exists an $n$-th root $\mu_{n} \in M^{1}(X, \omega)$ with the property that $\mu_{n}^{n}=\mu$.

The set of all infinitely divisible measures in $M^{1}(X, \omega)$ will be abbreviated by $I(X, \omega)$.

5.2 Theorem. For every $\mu \in I(X, \omega)$ the following statements are equivalent:

(i) $\mu$ lacks idempotent factors.

(ii) $\mu * \phi\left(x_{0}\right) \neq 0$ for all $\phi \in Z$.

Proof. 1. (i) $\Rightarrow$ (ii). Let $\mu \in I(X, \omega)$ with $D(\mu)=\left\{1_{x_{0}}\right\}$, and let

$$
A:=\left\{\phi \in Z: \mu * \phi\left(x_{0}\right) \neq 0\right\} .
$$

For every $n \geqq 1$ there exists $\mu_{n} \in M^{1}(X, \omega)$ such that $\mu_{n}^{n}=\mu$. In particular

$$
\mu * \phi\left(x_{0}\right)=\left(\mu_{n} * \phi\left(x_{0}\right)\right)^{n}
$$

and hence

$$
\lim _{n \rightarrow \infty} \mu_{n} * \phi\left(x_{0}\right)=1_{A}(\phi)
$$

for all $\phi \in Z$. Since $1_{A}$ is continuous in $\phi_{0} \in Z$ and $(G, K)$ admits (GP), an application of Lévy's continuity theorem yields the existence of a measure $\nu \in M^{1}(X, \omega)$ with

$$
\lim _{n \rightarrow \infty} \mu_{n}(x)=\nu(x)
$$

for all $x \in X$. But $(G, K)$ is a discrete pair, whence 


$$
\lim _{n \rightarrow \infty} \mu_{n} * \phi\left(x_{0}\right)=\nu * \phi\left(x_{0}\right)
$$

and thus $1_{A}(\phi)=\mu * \phi\left(x_{0}\right)$ for all $\phi \in Z$. The idempotency of $\nu$ follows from the following inequalities valid for all $\phi \in Z$ :

$$
\begin{aligned}
\nu * \nu * \phi\left(x_{0}\right) & =\nu * \phi\left(x_{0}\right) \nu * \phi\left(x_{0}\right) \\
& =1_{A}(\phi) 1_{A}(\phi) \\
& =1_{A}(\phi) \\
& =\nu * \phi\left(x_{0}\right) .
\end{aligned}
$$

Analoguously we obtain that $\nu \mid \mu$. But by assumption we have that $D(\mu)=\left\{1_{x_{0}}\right\}$, hence $\nu=1_{x_{0}}$ and thus $A=Z$.

2. (ii) $\Rightarrow$ (i). Let $\mu \in M^{1}(X, \omega)$ with $\mu * \phi\left(x_{0}\right) \neq 0$ for all $\phi \in Z$ and let $\lambda \in D(\mu)$. Then

$$
\mu * \phi\left(x_{0}\right)=\mu * \lambda * \phi\left(x_{0}\right)=\mu * \phi\left(x_{0}\right) \lambda * \phi\left(x_{0}\right),
$$

hence

$$
\lambda * \phi\left(x_{0}\right)=1=1_{x_{0}} * \phi\left(x_{0}\right)
$$

for all $\phi \in Z$ and thus $\lambda=1_{x_{0}}$.

From now on we suppose that $(G, K)$ is a symmetric discrete Gelfand pair.

5.3 THEOREM. For every measure $\mu \in I(X, \omega)$ lacking an idempotent factor there exist a measure $\nu \in M^{1}(X, \omega)$ and a number $\alpha>0$ satisfying

$$
\mu * \phi\left(x_{0}\right)=\exp \left[\alpha\left(\nu * \phi\left(x_{0}\right)-1\right)\right]
$$

for all $\phi \in Z$.

Proof. By hypothesis, for the given $\mu \in I(X, \omega)$ and every $n \geqq 1$ there is $a \mu_{n} \in M^{1}(X, \omega)$ such that $\mu_{n}^{n}=\mu$. Since $(G, K)$ is a symmetric pair, $\mu * \phi\left(x_{0}\right) \in \boldsymbol{R}$ for all $\phi \in Z$. The mapping $\phi \rightarrow \mu * \phi\left(x_{0}\right)$ from $Z$ into $\boldsymbol{R}$ is continuous on the compact space $Z$, hence there exists a $\phi_{1} \in Z$ such that

$$
\mu * \phi_{1}\left(x_{0}\right) \leqq \mu * \phi\left(x_{0}\right)
$$

for all $\phi \in Z$. We now apply Proposition 3.3 which yields

$$
\begin{aligned}
\mu * \phi_{1}\left(x_{0}\right) & =\int \mu * \phi_{1}\left(x_{0}\right) \check{\phi}\left(x_{0}\right) \sigma(d \phi) \\
& \leqq \int \mu * \phi\left(x_{0}\right) \check{\phi}\left(x_{0}\right) \sigma(d \phi) \\
& =\mu\left(x_{0}\right) .
\end{aligned}
$$


$\mu$ was assumed to lack idempotent factors. By Theorem 5.2 we therefore have

$$
0<\left(\mu_{2} * \phi\left(x_{0}\right)\right)^{2}=\mu * \phi\left(x_{0}\right)
$$

whenever $\phi \in Z$. We put

$$
\alpha:=-\log \mu * \phi_{1}\left(x_{0}\right)
$$

and for every $n \geqq 1$,

$$
\nu_{n}:=\frac{\mu_{n}-\exp \left(-\frac{\alpha}{n}\right) 1_{x_{0}}}{1-\exp \left(-\frac{\alpha}{n}\right)} .
$$

Clearly, $\alpha$ is well-defined. Moreover for every $n \geqq 1, \nu_{n} \in M^{1}(X, \omega)$. In fact $\nu_{n}(x) \geqq 0$ for all $x \in X \backslash\left\{x_{0}\right\}$, and since

$$
\mu\left(x_{0}\right) \geqq \mu * \phi_{1}\left(x_{0}\right),
$$

also $\nu_{n}\left(x_{0}\right) \geqq 0$. The fact that $\left\|\nu_{n}\right\|=1$ follows from $\left\|\mu_{n}\right\|_{1}=1$.

An easy computation shows that for all $n \geqq 1$ and $\phi \in Z$,

$$
\alpha+\log \mu * \phi\left(x_{0}\right)=n \log \left[\left(\exp \left(\frac{\alpha}{n}\right)-1\right) \nu_{n} * \phi\left(x_{0}\right)+1\right],
$$

with existing limit for $n \rightarrow \infty$. Consequently

$$
\alpha+\log \mu * \phi\left(x_{0}\right)=\alpha \lim _{n \rightarrow \infty} \nu_{n} * \phi\left(x_{0}\right)
$$

for all $\phi \in Z$. The mapping

$$
\phi \rightarrow \frac{1}{\alpha}\left(\alpha+\log \mu * \phi\left(x_{0}\right)\right)
$$

from $Z$ into $R$ is continuous in $\phi_{0}$. By Lévy's theorem there exists a measure $\nu \in M^{1}(X, \omega)$ satisfying

$$
\lim _{n \rightarrow \infty} \nu_{n}(x)=\nu(x)
$$

for all $x \in X$, and since $(G, K)$ is discrete, also

$$
\lim _{n \rightarrow \infty} \nu_{n} * \phi\left(x_{0}\right)=\nu * \phi\left(x_{0}\right)
$$

for all $\phi \in Z$. Altogether we obtain

$$
\alpha+\log \mu * \phi\left(x_{0}\right)=\alpha \nu * \phi\left(x_{0}\right)
$$


for all $\phi \in Z$, and the assertion follows.

5.4 Theorem. Let $\mu \in I(X, \omega)$ and $\lambda \in D(\mu)$ be the maximal idempotent factor of $\mu$. Then there exist a measure $\nu \in M^{1}(X, \omega)$ and a number $\alpha>0$ such that for all $\phi \in Z$

$$
\mu * \phi\left(x_{0}\right)= \begin{cases}\exp \left[\alpha\left(\nu * \phi\left(x_{0}\right)-1\right)\right] & \text { if } \lambda * \phi\left(x_{0}\right) \neq 0 \\ 0 & \text { otherwise. }\end{cases}
$$

Proof. Let $\mu$ and $\lambda$ be chosen according to the assumption. From Theorem 4.1 we infer the existence of a compact subgroup $K_{\lambda}$ of $G$ with the properties (ii) and (iii) of that theorem. Since $\mu * \lambda=\mu$, we have that $\mu \in A_{\lambda}$ and by Theorem 4.6 that $\tilde{\mu} \in M^{1}\left(X_{\lambda}, \omega_{\lambda}\right)$. We first show that

1. $\tilde{\mu}$ lacks idempotent factors.

Let $\tilde{\rho} \in D(\tilde{\mu})$. From Formula 4.4 we obtain that $\rho \in D(\mu)$. Since $\lambda$ is the maximal idempotent with $\lambda \mid \mu$, we have $\lambda=\lambda * \rho$, whence again by Formula $4.4, \tilde{\lambda}=\tilde{\lambda} *_{\lambda} \tilde{\rho}$. But $\tilde{\lambda}$ is the unit element of the Banach algebra $M^{1}\left(X_{\lambda}, \omega_{\lambda}\right)$ and so is $\tilde{\rho}$, which proves the assertion.

We note that Theorem 5.2 implies that

$$
\tilde{\mu} *_{\lambda} \tilde{\phi}\left(x_{\lambda}\left(T_{\lambda}, T_{\lambda}\right)\right) \neq 0
$$

for all $\tilde{\phi} \in Z_{\lambda}:=Z\left(X_{\lambda}, K_{\lambda}\right)$.

Next we show that

2. $\tilde{\mu} \in I\left(X_{\lambda}, \omega_{\lambda}\right)$ (defined analoguous to $I(X, \omega)$ ).

For every $n \geqq 1$ there exists $\mu_{n} \in M^{1}(X, \omega)$ such that $\mu_{n}^{n}=\mu$. Let $\phi \in Z$ be given with $\lambda * \phi\left(x_{0}\right)=0$. From $\mu=\lambda * \mu$ follows that $\mu * \phi\left(x_{0}\right)=0$, whence $\mu_{n} * \phi\left(x_{0}\right)=0$ and thus

$$
\mu_{n} * \lambda * \phi\left(x_{0}\right)=0=\mu_{n} *_{n} \phi\left(x_{0}\right)
$$

for all $n \geqq 1$. If on the other hand we pick a $\phi \in Z$ with $\lambda * \phi(x) \neq 0$, then from the idempotency of $\lambda$ follows $\lambda * \phi\left(x_{0}\right)=1$, and again we obtain

$$
\begin{aligned}
\mu_{n} * \lambda * \phi\left(x_{0}\right) & =\mu_{n} * \phi\left(x_{0}\right) \lambda * \phi\left(x_{0}\right) \\
& =\mu_{n} * \phi\left(x_{0}\right)
\end{aligned}
$$

for all $n \geqq 1$. But then Proposition 3.3 implies $\mu_{n} * \lambda=\mu_{n}$ and therefore $\mu_{n} \in A_{2}$ for all $n \geqq 1$. Theorem 4.6 and Formula 4.4 yield the assertion.

The main statement is a consequence of Theorem 5.3. At first we note that $\left(G, K_{\lambda}\right)$ admits $(\mathrm{GP})$, since $(G, K)$ does, so that Theorem 5.3 is applicable: There exist a measure $\tilde{\nu} \in M^{1}\left(X_{\imath}, \omega_{\lambda}\right)$ and a number $\alpha>0$ such 
that for all $\tilde{\phi} \in Z_{\lambda}$,

$$
\tilde{\mu} *_{\lambda} \tilde{\phi}\left(x_{\lambda}\left(T_{\lambda}, T_{\lambda}\right)\right)=\exp \left[\alpha\left(\tilde{\nu} *_{\lambda} \tilde{\phi}\left(x_{\lambda}\left(T_{\lambda}, T_{\lambda}\right)\right)-1\right)\right]
$$

Again appealing to Theorem 4.6 and to Formula 4.4 yields

$$
\mu *\left(x_{0}\right)=\exp \left[\alpha\left(\nu * \phi\left(x_{0}\right)-1\right)\right]
$$

valid for all $\phi \in A_{\lambda} \cap Z$. If there is a $\phi \in Z$ with $\lambda * \phi\left(x_{0}\right) \neq 0$, then for such a $\phi$ we obtain $\lambda * \phi\left(x_{0}\right)=1$ and by Proposition $3.1, \phi \in A_{\lambda}$.

5.5 Corollary. Let $\mu \in I(X, \omega)$. There exist a measure $\nu \in M^{1}(X, \omega)$ and a number $\alpha>0$ such that for all $\phi \in Z$ with $\mu * \phi\left(x_{0}\right) \neq 0$ we have the representation

$$
\mu * \phi\left(x_{0}\right)=\exp \left[\alpha\left(\nu * \phi\left(x_{0}\right)-1\right)\right] .
$$

Proof. Given $\mu \in I(X, \omega)$ there exists by Remark 4.8.2 a maximal idempotent factor $\lambda \in D(\mu)$. For all $\phi \in Z$ satisfying $\mu * \phi\left(x_{0}\right) \neq 0$ we then get

$$
\begin{aligned}
0 \neq \mu * \phi\left(x_{0}\right) & =\mu * \lambda * \phi\left(x_{0}\right) \\
& =\mu * \lambda\left(x_{0}\right) \mu * \phi\left(x_{0}\right),
\end{aligned}
$$

hence $\lambda * \phi\left(x_{0}\right) \neq 0$, and the theorem implies the assertion.

\section{§6. The Gelfand pair of the $m$-cube}

For an application of the Poisson type representation given in the preceding section we shall discuss the special case of the $m$-dimensional cube. In order to identify all infinitely divisible probability measures on the cube we need only exhibit the idempotents. For this task to be achieved the spherical functions on the cube have to be characterized.

\subsection{The $\boldsymbol{m}$-cube as a finite Gelfand pair}

Let $m \geqq 1$ and let $T$ denote the set of vertices of a regular polytope in $\boldsymbol{R}^{m}$. For all $(s, t) \in T \times T$ we define $d(s, t)$ to be the minimal number of vertices to be passed in moving along the edges from $s$ to $t$, where $t$ is counted but not $s$ and $s \neq t$. If $s=t$ we put $d(s, t):=0$. Clearly, $d$ is a metric on $T$. The set $G$ of all bijective mappings $T \rightarrow T$ which preserve the distance with respect to $d$, forms a group, and under the mapping

$$
(g, t) \rightarrow g(t)
$$

from $G \times T$ into $T,(G, T)$ becomes a homogeneous space. Given $t_{0} \in T$, 
the stabilizer $K$ of $t_{0}$ and the double coset space $X$ of $(G, T)$ we see that for $(s, t)$ and $\left(s^{\prime}, t^{\prime}\right) \in T \times T$

$$
\begin{aligned}
x(s, t)=x\left(s^{\prime}, t\right) & \Leftrightarrow \text { There is a } g \in G \text { with } g(s)=s^{\prime} \text { and } g(t)=t^{\prime} \\
& \Leftrightarrow d(s, t)=d\left(s^{\prime}, g^{\prime}\right) .
\end{aligned}
$$

Since $d(s, t)=d(t, s)$, we obtain that

$$
x(s, t)=x(t, s)
$$

for all $(s, t) \in T \times T$, and hence $(G, K)$ is a symmetric (finite) Gelfand pair. Specifying $T$ to be the set

$$
\left\{t=\left(t^{1}, \cdots, t^{m}\right) \in \boldsymbol{R}^{m}: t^{i}= \pm 1 \text { for all } i=0, \cdots, m\right\}
$$

of vertices of the $m$-dimensional cube and $t_{0}:=(1, \cdots, 1) \in T$ we obtain the Gelfand pair $(G, K)$ of the m-dimensional cube. It is clear that in this particular case the metric $d$ takes values in the set $\{0,1, \cdots, m\}$, hence $X=\left\{x_{0}, \cdots, x_{m}\right\}$ with

$$
x_{j}:=\{(s, t) \in T \times T: d(s, t)=j\}
$$

whenever $j=0, \cdots, m$. In particular $x_{0}=x(t, t)$ for all $t \in T$. We also note that the measure $\omega$ on $X$ is given by

$$
\omega\left(x_{j}\right)=\left(\begin{array}{c}
m \\
j
\end{array}\right)
$$

for all $j=0,1, \cdots, m$.

6.1.1. The convolution in $L^{1}(X, \omega)$ is given in terms of

$$
1_{x_{i}} 1_{x_{j}}\left(x_{k}\right)=\sum_{i=0}^{k}\left(\begin{array}{c}
k \\
l
\end{array}\right)\left(\begin{array}{c}
m-k \\
i-l
\end{array}\right) \delta_{i+l-2, j}
$$

for $x_{i}, x_{j}, x_{k} \in X$.

6.1.2. The spherical functions of $(G, K)$ can be computed as follows: For all $i=0,1, \cdots, m$ we introduce functions $\phi_{i}$ on $X$ implicitly by the expression

$$
\sum_{j=0}^{m} \phi_{i}\left(x_{j}\right) \omega\left(x_{j}\right) z^{j}=(1-z)^{i}(1+z)^{m-i}
$$

valid for all $z \in R$, or explicitly by

$$
\phi_{i}\left(x_{j}\right) \omega\left(x_{j}\right)=\sum_{l=0}^{j}(-1)^{l}\left(\begin{array}{l}
i \\
l
\end{array}\right)\left(\begin{array}{c}
m-i \\
j-l
\end{array}\right)
$$


for all $j=0,1, \cdots, m$. The following properties can be verified

(i ) $\left\{\phi_{i}: i=0,1, \cdots, m\right\}=S:=S(G, K)$.

(ii) $\phi_{i}\left(x_{j}\right)=\phi_{j}\left(x_{i}\right)$ for all $i, j=0,1, \cdots, m$.

(iii) $\left\langle\phi_{i}, \phi_{j}\right\rangle=\frac{2^{m}}{\omega\left(x_{i}\right)} \delta_{i j}$ for all $i, j=0,1, \cdots, m$.

(iv) The family

$$
\left\{\frac{1}{\sqrt{\left\langle\phi_{i}, \phi_{j}\right\rangle}} \phi: i=0,1, \cdots, m\right\}
$$

is a basis of the Hilbert space $L^{1}(X, \omega)$.

\subsection{The idempotents in $M^{1}(X, \omega)$}

6.2.1. There exist at least two idempotents in $M^{1}(X, \omega)$, namely the functions $1_{x_{0}}$ and $\left(\sum_{x \in X} \omega(x)\right)^{-1} 1_{X}$.

6.2.2. For every $\lambda \in M^{1}(X, \omega)$ the following statements are equivalent:

(i) $\lambda$ is an idempotent.

(ii) For every $\phi \in S$ either $\langle\lambda, \phi\rangle=1$ or $\langle\lambda, \phi\rangle=0$ holds.

Indeed, if $\lambda \in M^{1}(X, \omega)$ is an idempotent, i.e. $\lambda * \lambda=\lambda$, then

$$
\langle\lambda, \phi\rangle\langle\lambda, \phi\rangle=\langle\lambda * \lambda ; \phi\rangle=\langle\lambda, \phi\rangle
$$

for all $\phi \in S$, whence (ii). If, on the other hand, $\lambda \in M^{1}(X, \omega)$ satisfies (ii) then by (2) of Proposition 3.2 we have

$$
\begin{aligned}
\lambda & =\sum_{\phi \in S} \frac{\langle\lambda, \phi\rangle}{\langle\phi, \phi\rangle} \cdot \phi \\
& =\sum_{\phi \in S} \frac{\langle\lambda, \phi\rangle\langle\lambda, \phi\rangle}{\langle\phi, \phi\rangle} \phi \\
& =\sum_{\phi \in S} \frac{\langle\lambda * \lambda, \phi\rangle}{\langle\phi, \phi\rangle} \phi \\
& =\lambda * \lambda, \text { i.e. (i). }
\end{aligned}
$$

6.2.3. If $\lambda \in M^{1}(X, \omega)$ is an idempotent then we have for every $x \in X$ and each $\phi \in S$ that $\lambda(x)>0$ and $\langle\lambda, \phi\rangle=1$ imply $\phi(x)=1$.

Indeed, for the given $\lambda \in M^{1}(X, \omega)$ fulfilling the hypotheses we obtain

$$
\sum_{x \in X} \lambda(x) \omega(x)=1=\langle\lambda, \phi\rangle=\sum_{x \in X} \lambda(x) \check{\phi}(x) \omega(x)=\sum_{x \in X} \lambda(x) \phi(x) \omega(x)
$$

and thus

$$
\sum_{x \in X} \lambda(x) \omega(x)(1-\phi(x))=0 .
$$

Since $|\phi(x)| \leqq 1$ for all $x \in X$, the assertion follows. 
6.2.4. Let $i, j \in\{0,1, \cdots, m\}$. The following statements are equivalent:

(i) $\phi_{i}\left(x_{j}\right)=1$.

(ii) We have either $i=0$ or $j=0$, or we have $i=m$ and $j$ even, or we have $j=0$ and $i$ even.

(ii) $\Rightarrow$ (i) follows directly from the equality

$$
\left(\begin{array}{c}
m \\
j
\end{array}\right) \phi_{i}\left(x_{j}\right)=\sum_{l=0}^{j}(-1)^{l}\left(\begin{array}{l}
i \\
l
\end{array}\right)\left(\begin{array}{c}
m-i \\
j-l
\end{array}\right)
$$

valid for all $i, j \in\{0,1, \cdots, m\}$. It remains to show (i) $\Rightarrow$ (ii). For all $i, j \in\{0,1, \cdots, m\}$ such that $\phi_{i}\left(x_{j}\right)=1$ we get

$$
\begin{aligned}
\omega\left(x_{j}\right) \omega\left(x_{j}\right) & =1_{x_{j}} * 1_{x_{j}} * \phi_{i}\left(x_{0}\right) \\
& =\sum_{k=0}^{m} 1_{x_{j}} * 1_{x_{j}}\left(x_{k}\right) \phi_{i}\left(x_{k}\right) \omega\left(x_{k}\right) \\
& =\sum_{k=0}^{m} \sum_{l=0}^{k}\left(\begin{array}{c}
k \\
l
\end{array}\right)\left(\begin{array}{c}
m-k \\
j-l
\end{array}\right) \delta_{k-2 l, 0} \omega\left(x_{k}\right) \phi_{i}\left(x_{k}\right) \\
& =\sum_{l=0}^{j} \sum_{k=0}^{i}\left(\begin{array}{l}
j \\
l
\end{array}\right)\left(\begin{array}{c}
m-j \\
k-l
\end{array}\right) \delta_{k, 2 l} \omega\left(x_{j}\right) \phi_{i}\left(x_{k}\right) \\
& =\omega\left(x_{j}\right) \sum_{l=0}^{j}\left(\begin{array}{l}
j \\
l
\end{array}\right)\left(\begin{array}{c}
m-j \\
l
\end{array}\right) \phi_{i}(2 l)
\end{aligned}
$$

and since $\omega\left(x_{j}\right)=\sum_{l=0}^{j}\left(\begin{array}{l}j \\ l\end{array}\right)\left(\begin{array}{c}m-j \\ l\end{array}\right)$,

$$
0=\sum_{l=0}^{j}\left(\begin{array}{l}
j \\
l
\end{array}\right)\left(\begin{array}{c}
m-j \\
l
\end{array}\right)\left(1-\phi_{i}(2 l)\right)
$$

which implies $\phi_{i}(2 l)=1$ for all $l=0, \cdots, j$. Noting that

$$
\phi_{m}\left(x_{j}\right)=(-1)^{j}
$$

for all $x_{j} \in X$ we conclude the assertion.

6.2.5. There are exactly four idempotents in $M^{1}(X, \omega)$, namely the functions $1_{x_{0}}, 2^{-m} 1_{X}, \lambda_{0}$ defined by

$$
\lambda_{0}\left(x_{j}\right):= \begin{cases}2^{1-m} & \text { if } j \text { even } \\ 0 & \text { if } j \text { odd }\end{cases}
$$

and $\lambda_{1}$ defined by

$$
\lambda_{1}\left(x_{j}\right):= \begin{cases}\frac{1}{2} & \text { if } j=0 \\ 0 & \text { if } 0<j<m, \quad \text { and } \\ \frac{1}{2} & \text { if } j=m .\end{cases}
$$


At first we show that the functions $\lambda_{0}$ and $\lambda_{1}$ are in fact idempotents (the remaining ones clearly are). For every $j=0,1, \cdots, m$ we have

$$
\begin{aligned}
& \left\langle\lambda_{0}, \phi_{j}\right\rangle=2^{1-m} \sum_{i \text { even }} \phi_{j}\left(x_{i}\right) \omega\left(x_{i}\right) \\
& = \begin{cases}0 & \text { if } j=1, \cdots, m-1 \\
1 & \text { if } j=0, m .\end{cases}
\end{aligned}
$$

An application of 6.2 .2 shows that $\lambda_{0}$ is an idempotent. Analogously one deduces from

$$
\begin{aligned}
\left\langle\lambda_{1}, \phi_{j}\right\rangle & =\frac{1}{2} \phi_{j}\left(x_{0}\right)+\frac{1}{2} \phi_{j}\left(x_{m}\right)(-1)^{j} \\
& = \begin{cases}0 & \text { if } j \text { odd } \\
1 & \text { if } j \text { even }\end{cases}
\end{aligned}
$$

that $\lambda_{1}$ is also an idempotent.

Now we assume $\lambda \in M^{1}(X, \omega)$ to be an idempotent.

1) Let $j$ exist with $0<j<m$ and $\left\langle\lambda, \phi_{j}\right\rangle=1$. Then for each $i=0$, $1, \cdots, m$ with $\lambda\left(x_{i}\right)>0$ we obtain from 6.2.3 that $\phi_{j}\left(x_{i}\right)=1$ and from 6.2.4 that consequently $i=0$ or $i=m$. But this implies $\lambda=\lambda\left(x_{0}\right) 1_{x_{0}}$ or $\lambda=$ $\lambda\left(x_{0}\right) 1_{x_{0}}+\lambda\left(x_{0}\right) 1_{x_{m}}$. Since $\lambda$ is a probability measure, this implies that either $\lambda=1_{x_{0}}$ or $\lambda=\frac{1}{2} 1_{x_{0}}+\frac{1}{2} 1_{x_{m}}=\lambda_{1}$. Thus $\lambda$ is of the asserted form.

2) Let there exist no $j$ with $0<j<m$ and $\left\langle\lambda, \phi_{j}\right\rangle=1$.

2a) If $\left\langle\lambda, \phi_{m}\right\rangle=0$ then there is an $\alpha \in C$ such that $\lambda=\alpha \phi_{0}=\alpha 1_{X}$, and in this case therefore $\lambda=2^{-m} 1_{X}$.

2b) If $\left\langle\lambda, \phi_{m}\right\rangle=1$ then there are $\alpha, \beta \in C$ such that $\lambda=\alpha \phi_{0}+\beta \phi_{m}$. We obtain

$$
1=\left\langle\lambda, \phi_{m}\right\rangle=\beta\left\langle\phi_{m}, \phi_{m}\right\rangle=\beta 2^{m}
$$

and

$$
1=\left\langle\lambda, \phi_{0}\right\rangle=\alpha\left\langle\phi_{0}, \phi_{0}\right\rangle=\alpha 2^{m}
$$

whence

$$
\lambda=2^{-m} \phi_{0}+2^{-m} \phi_{m}
$$

Since $\phi_{m}\left(x_{j}\right)=(-1)^{j}$ for all $j=0,1, \cdots, m$, this implies that $\lambda=\lambda_{0}$.

From 1) and 2) follows that all idempotents in $M^{1}(X, \omega)$ are of the asserted form. 


\section{REFERENCES}

[1] S. Ben Mansoor, Le cube comme couple de Gelfand: Applications, probabiliste et généralisations, Thèse présentée à l'Université Paul Sabatier de Toulouse (1984), 64 pages.

[2] Ph. Bougerol, Un mini-cours sur les couples de Guelfand, Publications du Laboratoire de Statistique et Probabilités, Université Paul Sabatier, Toulouse, (Avril 1983).

[ 3 ] J. Dieudonné, Grundzüge der modernen Analysis, Band 5/6, Vieweg, BraunschweigWiesbaden 1979.

[4] L. Gallardo, O. Gebuhrer, Lois de probabilité infiniment divisibles sur les hypergroupes commutatifs, discrets, dénombrables, In: Probability Measures on Groups VII (edited by H. Heyer), 116-130. Proceedings, Oberwolfach, Germany (1983), Lecture Notes in Math., 1064, Springer, 1984.

[5] J. Gilewski, K. Urbanik, Generalized convolutions and generating functions, Bull. Acad. Sci. Polon. Ser. Math. Astr. Phys., 16 (1968), 481-487.

[6] H. Heyer, Probability measures on locally compact groups, Springer, BerlinHeidelberg-New York, 1977.

[7] - Convolution semigroups of probability measures on Gelfand pairs, Expo. Math., 1 (1983), 3-45.

[ 8 ] G. Letac, Problèmes classiques de probabilité sur un couple de Gelfand, In: Analytic Methods in Probability Theory (edited by D. Dugué, E. Lukacs, V. K. Rohatgi), 93-120, Proceedings, Oberwolfach, Germany (1980), Lecture Notes in Math., 861, Springer 1981.

[9] - Les fonctions sphériques d'un couple de Gelfand symétrique et les chaînes de Markov, Adv. Appl. Prob., 14 (1982), 272-294.

[10] G. Letac, L. Takács, Random walks on the $m$-dimensional cube, J. reine angew. Math. (Crelle), 310 (1979), 187-195.

[11] A. Schwartz, Generalized convolutions and positive definite functions associated with general orthogonal series, Pacific J. Math., 55,2 (1974), 565-582.

Universität Tübingen

Mathematisches Institut

Auf der Morgenstelle 10

7400 , Tübingen

Deutsche Bundesrepublik 